\title{
Traitement et valorisation des vases de dragages par solidification/stabilisation : état de l'art
}

BOUTOUIL M

Docteur, Université du Havre, Laboratoire de Mécanique

\section{LEVACHER D}

Professeur des Universités, Université de Caen, Centre de Géomorphologie, M2C UPRES A 6143 CNRS

\section{Résumé :}

Les traitements de sédiments contaminés sont nombreux et variés. Néanmoins, la tendance actuelle est au traitement privilégiant la valorisation par réutilisation. L'article présenté ici s'attache à établir un état des connaissances en matière du devenir actuel des sédiments, contaminés ou pas, de leurs traitements ainsi que de leur valorisation notamment par la technique de solidification/stabilisation $(\mathrm{S} / \mathrm{S})$ à base de ciment. Cette technique appliquée aux sédiments présente de multiples avantages : d'une part donner lieu à un produit solide (sachant que la teneur en eau des sédiments peut atteindre $200 \%$ ), et d'autre part, stabiliser et immobiliser les contaminants inorganiques.

A travers des exemples de $\mathrm{S} / \mathrm{S}$, on constate que le ciment est le liant le plus utilisé avec des additifs tels que les cendres volantes. Les différentes étapes menant au traitement et à la valorisation ainsi que les diverses formes de valorisation en techniques routières et en remblaiement sont présentées. On constate également une variété de procédés, dont les formulations dépendent essentiellement des caractéristiques géotechniques et chimiques du produit à traiter et de l'utilisation finale du matériau traité. L'objectif global est d'obtenir de meilleurs caractéristiques physico-mécaniques, un taux d'immobilisation des contaminants élevé, avec des dosages en ciment et additifs faibles, tout en s'efforçant de limiter les coûts de traitement.

\begin{abstract}
:
Despite wide range of treatments technique of contaminated sediments, the current trends encourage treatments that result in reuse of the treated material. This paper establishes a state of the art as regards alternative solutions to common deposit at sea and in land. The cement-based solidification/stabilisation (S/S) technique offers two main advantages that can help to overcome contaminated dredged material problems : First, the technique improves the physical and mechanical characteristics of the treated sediment, second, solidification/stabilisation reduces the mobility of inorganic contaminants (e.g. heavy metals).

This paper presents different S/S examples, which show that cement is the most used binder with additives such as fly ash. S/S process consists in different
\end{abstract}


steps in developing a composition that will meet the desired physical mechanical and environmental objectives and specifications for the minimum costs

\section{Introduction}

La navigation au sein des voies d'eau ou dans les zones portuaires nécessite un entretien régulier permettant aux navires de circuler sans risques. Les opérations de dragage ont pour but d'enlever les sédiments se déposant sur le fond et assurer ainsi un tirant d'eau minimum. A défaut, l'envasement peut prendre des proportions considérables, au point de ne plus autoriser la navigation (SPAQUE, 1998).

La gestion des sédiments de dragage, contaminé ou pas, doit ensuite faire face, de plus en plus, à maintes difficultés liées essentiellement à la raréfaction des sites de dépôts à terre et aux réglementations en matière de protection de l'environnement. Ceci sans oublier les répercussions de telles solutions sur l'environnement aquatique et terrestre dans le cas des sédiments contaminés. Cette gestion des matériaux dragués revêt dont un caractère d'importance.

Parmi les nombreuses techniques utilisées actuellement pour traiter les sédiments de dragage, la solidification/stabilication à base de liants hydrauliques offe de multiples avantages. Outre la stabilisation et l'immobilisation des contaminants inorganiques, la $\mathrm{S} / \mathrm{S}$ ouvre des perspectives de valorisation du matériau traité. Plusieurs études ainsi que des projets industriels, notamment au Canada (Stegemann et coll., 1991 ; Environnement Canada 1993), au Japon (Kamon et coll., 1990 ; Ogino et coll., 1994 ; Akinori, 1998), aux Etats-Unis (USEPA, 1998) et au Pays-Bas (Van Leeuwen et coll., 1997), montrent que cette technique est largement adoptée et utilisée.

Dans cet article, la technique de solidification/stabilisation ainsi que les différentes étapes qui mènent à la valorisation des produits traités seront discutées à la lumière de plusieurs exemples de $\mathrm{S} / \mathrm{S}$. Pour plus de détails concernant cette technique et ses différents aspects ainsi que les travaux de recherches menés sur la solidification/stabilisation d'un sédiment de dragage du Havre, les lecteurs se reporterons à la thèse (Boutouil, 1998).

\section{Les sédiments de dragage et leur devenir}

Que ce soit dans les ports de mer ou les ports d'estuaire, le bilan des dragages en France fait apparaître une quantité considérable de matériaux dragués. On estime que 50 millions de $\mathrm{m}^{3}$ de sédiments sont dragués chaque année (Géode, 1994 ; Alzieu, 1999). Sur l'ensemble des matériaux dragués, les vases représentent environ $75 \%$. A l'étranger, les chiffres relatifs aux quantités draguées indiquent des volumes élevés : $40,45,50,250$ millions de $\mathrm{m}^{3}$ par an, respectivement pour le Royaume Uni, les Pays Bas, l'Allemagne et les Etats Unis (Boutouil, 1998).

Le devenir et l'évacuation des sédiments dragués constituent une partie tout aussi importante du cycle de dragage et dépendent de leurs caractéristiques géotechniques (granulométrie, teneur en eau...) et de leurs qualités (contamination). En France, le degré de contamination des sédiments de dragage destinés à l'immersion est apprécié par rapport à des valeurs de référence (Tableau 
1) définis par le groupe d'étude et d'observation sur le dragage et l'environnement (Géode). La définition des ces valeurs a été basée sur une étude statistique (Géode, 1994) et sur la convention d'Oslo qui défini les deux niveaux suivants :

Niveau 1 : au-dessous de ce premier niveau, l'immersion des matériaux de dragage serait autorisée sans études complémentaires.

Niveau 2 : au-dessus de ce niveau, l'immersion est susceptible d'être interdite sous réserve que l'interdiction soit le moins dommageable pour l'environnement.

\begin{tabular}{|l|c|c|c|}
\hline $\begin{array}{l}\text { Contaminants } \\
\text { (mg/kg sec) }\end{array}$ & BdF $^{*}$ & Niveau 1 & Niveau 2 \\
\hline Arsenic & 4.4 & 25 & 50 \\
\hline Cadmium & 0.5 & 1.2 & 2.4 \\
\hline Chrome & 45 & 90 & 180 \\
\hline Cuivre & 35 & 45 & 90 \\
\hline Mercure & 0.2 & 0.4 & 0.8 \\
\hline Nickel & 20 & 37 & 74 \\
\hline Plomb & 47 & 100 & 200 \\
\hline Zinc & 115 & 276 & 552 \\
\hline
\end{tabular}

* : Bruit de Fond concentrations en métaux considérées comme naturelles pour les sédiments portuaires

\section{Tableau 1 : Niveaux de références pour les métaux lourds. Géode (1996)}

Quant aux modes d'évacuation des matériaux de dragage, on en distingue deux actuellement : d'une part le dépôt à terre et d'autre part l'immersion en mer. En ce qui concerne le dépôt à terre, c'était l'une des options les plus retenues. Bien que cette solution présente l'avantage de la valorisation ultérieure, elle se heurte à plusieurs difficultés : manque de sites adéquats, inexploitation du site le temps de la consolidation et du tassement, risque de contamination de la nappe phréatique par infiltration, solubilisation des métaux lourds et contamination de l'environnement terrestre (Environnement Canada, 1994).

L'immersion en mer constitue l'autre mode d'évacuation des matériaux de dragage couramment adopté. Néanmoins, une partie des matériaux évacués par voie de mer peut retourner vers l'endroit où elle a été draguée à cause des courants marins (A.I.P.C.N., 1990). Lorsque les sédiments sont contaminés, l'une des méthodes souvent envisagée et retenue pour les gérer est le confinement en milieu aquatique (capping). Cette technique consiste à placer les sédiments contaminés dans un site où les conditions hydrodynamiques assurent la stabilité des matériaux, et à les couvrir d'une couche de sable ou de vase non contaminée d'une épaisseur variant de moins de un mètre à plus de deux mètres. Bien que le recouvrement en milieu aquatique soit une solution plus intéressante (peu onéreuse) et plus efficace que le dépôt à terre, cette solution soulève plusieurs préoccupations environnementales. Elles concernent notamment le risque de migration des sédiments à travers la couche protectrice ainsi que la durée pendant laquelle la couverture pourrait isoler efficacement les produits chimiques toxiques de l'environnement marin. 
On le constate, la gestion des sédiments de dragage passe par l'appréciation de l'impact potentiel de l'immersion et de la mise en dépôt des sédiments contaminés sur l'environnement récepteur ainsi que par la recherche de solutions alternatives de traitement. La technique de solidification/stabilisation à base de liants hydrauliques présente un potentiel de traitement largement démontré. Elle permet en effet de réduire la mobilité des contaminants inorganiques et de produire un matériau pouvant être valorisé.

\section{La technique de solidification/stabilisation}

\subsection{Description de la technique :}

La technique de solidification/stabilisation est bien répandue dans le domaine du traitement des boues industrielles et des boues des stations d'épuration. Son utilisation pour les sols et sédiments contaminés est en voie de développement (Boutouil, 1998).

- Le processus de solidification se réfère à l'amélioration des caractéristiques physiques du produit final.

- Tandis que la stabilisaíion se rapporte au "piégeage" physique et chimique des éléments toxiques, notamment les métaux lourds, et à la réduction de leur mobilité, et ce grâce à la matrice solide formée par solidification.

$\mathrm{Par}$ conséquent, les objectifs fondamentaux du traitement par S/S sont la réduction de l'impact négatif de la contamination sur l'environnement ainsi que l'obtention d'un produit ayant une intégrité structurelle. Les technologies de S/S en utilisation inchuent un large éventail de liants : ciment, chaux, polymère, matériaux pouzzolaniques..., avec ajout d'additifs tels que : cendres volantes, laitiers... Néanmoins le ciment reste le liant le plus utilisé dans la technique de solidification/stabilisation. L'ensemble du processus de solidification/stabilisation est obtenu grâce à l'hydratation des constituants du ciment. La pâte, résultant du mélange du ciment anhydre (avec d'éventuels ajouts) et de l'eau de gâchage issue du produit à traiter ou volontairement rajouter, va faire prise et durcir.

\subsection{Procédés et exemples de $\mathrm{S} / \mathrm{S}$ :}

La solidification/stabilisation trouve son utilisation autant dans le domaine des sols que dans celui des sédiments contaminés ou non. D'après les recherches bibliographiques menées dans le cadre de la thèse (Boutouil, 1998), on note une variété de procédés qui dépendent essentiellement des caractéristiques géotechniques et chimiques du produit à traiter et de l'utilisation finale du matériau traité. L'objectif global est d'obtenir de meilleurs caractéristiques physico-mécaniques, un taux d'immobilisation des contaminants élevé, avec des dosages en ciment et additifs faibles tout en s'efforçant de limiter les coûts de traitement.

Рar ailleurs, l'ensemble des techniques de solidification/stabilisation présente un certain nombre d'étapes communes à respecter :

- Analyse et évaluation du produit à traiter : caractérisation. 
- Prétraitement éventuel : baisse de la teneur en eau.

- Addition des liants et des agents solidifiants puis mélange : in-situ ou ex-situ.

- Compactage éventuel.

- Contrôle de qualité du matériau final.

- Dépôt ou utilisation du matériau final.

Voici à présent des exemples de procédés de solidification/stabilisation qui se situent à divers stades de développement : étude de recherche, étude de faisabilité, projet pilote et réalisation de chantier.

\subsubsection{Les sédiments solidifiés/stabilisés}

Dans l'étude présentée par Kamon et coll. (1990), le processus de solidification de la vase a été réalisé à l'aide de deux ciments : un ciment Portland (OPC) et un ciment avec ajout de $20 \%$ de laitiers d'acier inox. Cette étude s'inscrit dans l'optique de valorisation de deux matériaux : un déchet industriel (les laitiers d'acier inox) et une vase de dragage. Les dosages en ciment étaient de $6,9,12$ et $15 \%$ de la masse de la vase et chacun de ces mélanges a été soumis à la résistance à la compression et à l'essai CBR. La conservation des éprouvettes cylindriques s'est faite sans perte d'humidité. Cette condition de conservation a été également adoptée dans le cadre du programme de recherche rapporté par Stegemann et coll. 1991. Après solidification/stabilisation du sédiment de dragage selon des procédés propres à des industriels participant au programme, la maturation des éprouvettes cubiques s'est effectuée dans leurs moules pendant une période minimale de 56 jours. Seules deux entreprises ont consenti à fournir le descriptif de leurs procédés de solidification/stabilisation. On notera en particulier (Tableau 2) que ces compositions emploient plusieurs additifs dans des proportions variables, ce qui montre que les formulations doivent être optimisées afin d'obtenir un produit solidifié/stabilisé qui offre les meilleures caractéristiques de résistance à l'altération par dégradation physique et par lixiviation.

\begin{tabular}{|c|c|c|}
\hline Nom de la compagnie & Dansk Beton Teknik A/S & Tricil ltd \\
\hline $\begin{array}{l}\text { Traitement du déchet avant } \\
\text { solidification }\end{array}$ & Non & Non \\
\hline Masse du déchet solidifié & $8265 \mathrm{~g}$ & $8000 \mathrm{~g}$ \\
\hline Additifs (g) : & $\begin{array}{l}\text { 1- Matériaux poreux : } 10657 \\
\text { 2- Ciment : } 2044 \\
\text { 3- Micro-matière de charge }: 545 \\
\text { 4- Sable : } 3736 \\
\text { 5- Eau et plastifiant : } 1000 \\
\end{array}$ & $\begin{array}{l}\text { 1- Acide chlorhydrique : } 2.4 \mathrm{~L} \\
\text { 2- Scories : } 480 \\
\text { 3- Chaux : } 900 \\
\text { 4- Scories : } 2500 \\
\text { 5- Ciment : } 750\end{array}$ \\
\hline Ordre d'addition & $\begin{array}{l}\text { Le matériau poreux a été ajouté } \\
\text { en premier }\end{array}$ & \multirow[t]{2}{*}{$(1+2+3) 1$ heure, $(4+5) 0.5 \mathrm{~h}$} \\
\hline Temps de mélange & 5 min. & \\
\hline
\end{tabular}

Tableau 2 : Fiches d'information fournies par deux entreprises canadiennes sur les procédés de solidification/stabilisation de sédiments de dragage. Stegemann et coll (1991) 
En examinant le projet de solidification d'une vase de dragage rapporté par Knox et coll. (1994), on constate que plusieurs agents solidifiants ont été étudiés afin de choisir le plus adapté. Les liants en question sont : du ciment (type non précisé), de la poussière ou scories des fours à ciment, de la chaux vive ou éteinte et un mélange ciment-chaux-cendres volantes (proportion 1:6:13). Les dosages de ces liants vont jusqu'à $50 \%$ de la masse humide de la vase avec un intervalle de $5 \%$. Les objectifs de ce travail sont d'une part d'évaluer l'effet du remaniement du produit solidifié sur la résistance à la compression. En effet, si le matériau traité est transporté vers un lieu de dépôt, ce qui est le cas de la $\mathrm{S} / \mathrm{S}$ ex-situ, le remaniement aura pour conséquence la rupture des liens mécaniques et par la suite une chute de la résistance. Et d'autre part, de développer une corrélation entre la résistance à la compression mesurée au laboratoire et le pénétromètre de "poche" utilisable sur site.

Egalement dans le domaine de revalorisation des sédiments de dragage par solidification et avec réalisation sur le terrain, le projet évoqué par Ogino et coll. (1994) vise à réutiliser les sédiments dragués dans une rivière puis solidifiés à des fins de remblaiement d'un quai constitué de palplanches métalliques. L'étude de faisabilité en laboratoire a consisté à tester trois types de ciment : un ciment Portland Normal (OPC), un ciment Portland aux laitiers de haut fourneau et un ciment appelé géo-ciment qui a été conçu pour stabiliser les sols à fortes teneurs en matières organiques. Les dosages initialement utilisés vont de 100 à $220 \mathrm{~kg} / \mathrm{m}^{3}$ afin d'évaluer les performances de chaque ciment vis-à-vis de la résistance à la compression.

\subsubsection{Les sols solidifiés/stabilisés}

Van Leeuwen et coll. (1997) indiquent que le travail s'inscrit dans le cadre d'une étude sur la faisabilité de la solidification/stabilisation à base de ciment appliquée aux sols contaminés. En effet, une surface de $1000 \mathrm{~m}^{2}$ a été recouverte d'une couche de sol contaminé (principalement par le cuivre, le plomb et le zinc) qui a été traité in-situ avec du ciment Portland. Les dosages en ciment (type non précisé) ont varié de 12 à $22 \%$ (par rapport à la masse du sol sec) en fonction de la teneur en eau initiale des échantillons de sol : le rapport $E / C$ étant pris aux environs de 1.

Au Canada, le sol du site Pacific Place contaminé par de fortes teneurs en métaux et de faibles teneurs en matières organiques (Environnement Canada, 1993) a fait l'objet de plusieurs études de faisabilité de la part d'entreprises spécialisées dans le traitement par solidification/stabilisation. Et ce dans le cadre du programme DELTAC (développement et démonstration de techniques d'assainissement de lieux contaminés). D'après les travaux réalisés, on note que la démarche adoptée par l'ensemble des entreprises participantes, consiste à préparer plusieurs'formulations puis à en choisir une, suite à des essais préliminaires de résistance à la compression ou de lixiviation. La destination finale du sol traité, à savoir : une utilisation ou une élimination en décharge, est fonction des résultats du protocole d'essais suivant :

- Dosage du carbone organique total (COT \%).

- Extraction à l'équilibre (lixiviation d'échantillon broyé pendant 7 jours). 
- Extraction chimique séquentielle (lixiviation d'échantillon broyé).

- Essai de lixiviation dynamique (lixiviation d'échantillon monolithique).

- Détermination de la conductivité hydraulique $(\mathrm{m} / \mathrm{s})$.

- Résistance à la compression simple.

- Essais de gel-dégel et mouillage-séchage.

- Capacité de neutralisation des acides.

L'ensemble de ces essais ainsi que leur programme d'étude ont été présentés et discutés dans le cadre de la thèse (Boutouil, 1998).

Le procédé présenté par la Chemical Waste Management of Canada, Inc. utilise, pour $10 \mathrm{~kg}$ de sol contaminé, de l'eau et du ciment Portland dans des proportions de 0.1 et de 0.25 respectivement. Les échantillons préparés ont été conservés dans des moules pendant 28 et 56 jours afin d'évaluer l'influence du temps de conservation puis soumis à la batterie d'essais indiqués ci-dessus. Sans donner plus de précisions, la Newalta Corporation indique que du ciment Portland et des cendres volantes ont été utilisés pour stabiliser/solidifier le sol contaminé dans des proportions de 1:1. La période de conservation des échantillons était de 56 jours. Une autre démarche a été adoptée par l'entreprise Bennett Remediation Services. Elle a consisté à incinérer le sol à $370{ }^{\circ} \mathrm{C}$ puis à le stabiliser/solidifier avec diverses préparations à base de ciment Portland, de cendres volantes et d'un aluminosilicate. Après des essais de lixiviation, deux préparations ont été retenues. Dans la première, le sol est mélangé à $10 \%$ de ciment tandis que dans la seconde, $2 \%$ d'aluminosilicate sont ajoutés aux $10 \%$ de ciment. D'autres entreprises utilisent, en plus du ciment Portland et des cendres volantes, des réactifs brevetés. C'est le cas de la Waste Stream Technology/Wastech, Inc. qui après 18 formulations, des essais de résistance à la compression et de lixiviation et une étude de coût, a choisi le mélange suivant : sol - eau et réactifs SuperSet ${ }^{\circledR}$ liant : 5-1-3.

\section{4- Condusions}

Les projets présentés dans cet article sont intéressants tant par la démarche adoptée visant à aboutir à la réutilisation du matériau extrait que par la preuve du potentiel de valorisation par application du principe de solidification/stabilisation. La démarche comporte les étapes suivantes :

- Caractérisation géotechnique et chimique du sédiment ou sol à traiter : teneur en eau, granulométrie, $\mathrm{pH}$, matières organiques, teneurs et nature des polluants ... Sachant que la $\mathrm{S} / \mathrm{S}$ n'est efficace que vis-à-vis des contaminants inorganiques (métaux lourds).

- La deuxième étape vise à répondre à la question cruciale : quels dosages en ciment et additifs permettent d'atteindre la résistance requise pour une telle utilisation et un taux d'immobilisation élevé, sans oublier les coûts du traitement. Lors de cette étape, plusieurs formulations sont proposées et testées en laboratoire puis optimisées en banc d'essai à l'aide d'essais préliminaires de résistance à la compression et de lixiviations. Les dosages en ciment semblent être de 10 à $20 \%$ ou de 130 à $260 \mathrm{~kg} / \mathrm{m}^{3}$. Quant aux coûts, l'USEPA (1998) rapporte qu'en dessous de $2.50 \mathrm{~m}$ de sol pollué, la $\mathrm{S} / \mathrm{S}$ ex-situ pourrait être 
moins cher que la $\mathrm{S} / \mathrm{S}$ in situ. Le coût de la $\mathrm{S} / \mathrm{S}$ in-situ varie de 25 à $50 \$ \mathrm{le} \mathrm{m}^{3}$ (125 à $250 \mathrm{FF}$ ), alors que le traitement ex-situ peut varier de 130 à $260 \$$ le $^{3}$ (650 à $1300 \mathrm{FF}$ ), dépendant du volume à traiter, de la structure du sol, la profondeur de traitement, le type de contaminants et des objectifs du traitement (résistance, immobilisation, perméabilité...)

- L'étape suivante consiste à réaliser un projet pilote ou en grandeur réelle puis prélever et tester des échantillons solidifiés/stabilisés afin de confirmer la formulation ainsi que les performances physico-mécaniques et environnementales spécifiées.

En ce qui concerne ces performances, les études (Boutouil, 1998) montrent que le comportement physico-mécanique à court terme est bien cerné, au contraire du long terme. Les études sur la durabilité, grâce à des essais de mouillage-séchage et gel-dégel par exemple, doivent en effet être intégrer dans les projets de valorisation par $\mathrm{S} / \mathrm{S}$. Quant au comportement environnemental, il doit être étudier en fonction des conditions physico-chimiques du site final de valorisation. Les recherches montrent que la mobilité des métaux lourds est influencée par ces conditions et que des métaux tels que le chrome (V), le cuivre, le mercure sont moins bien stabilisés.

D'après les exemples de solidification/stabilisation, on note également une diversité des méthodologies expérimentales et des formes de valorisation. Parmi les utilisations potentielles, les sous-couches routières et le remblaiement sont les plus couramment utilisées. La diversité des méthodologies montre que, pour plus d'efficacité, l'application de la solidification/stabilisation aux sols et sédiments, contaminés ou non, doit être réalisée au cas par cas.

\section{5-Références}

A.I.P.C.N. (1990) "Méthodes économiques d'entretien des chenaux". Supplément au Bulletin $N^{\circ} 69$, Rapport du Groupe de Travail $N^{\circ} 14,182 \mathrm{p}$.

Akinori S. (1998) "Cement and soft mud mixing technique using compressed airmixture pipeline : efficient solidification at a disposal site". WODCON WV, Las Vegas, USA, June 28-July 2, 15 p.

Alzieu C. (1999) "Dragages et environnement marin : état des connaissances". Edition IFREMER, 223 p.

Boutouil M. (1998) "Traitement des vases de dragage par solidification/stabilisation à base de ciment et additifs". Thèse de Doctorat, Université du Havre, Nov. 1998, 279 p. Publiée en livre. Edit. PARALIA ISBN 2-9505787-4-8

Environnement Canada (1993) "Projet de stabilisation/solidification, site de Pacific Place". Programme DETALC, Environnement Canada, N K1A OH3.

Environnement Canada (1993) "Projet de stabilisation/solidification, site de Pacific Place". Document Internet, http://www.doe.ca.

Environnement Canada (1994) "Répercussions environnementales du dragage et de la mise en dépôt des sédiments". Document préparé par Les Consultants Jacques Bérubé inc. Pour la Section du développement technologique. 
Direction de la protection de l'environnement, régions du Québec et de I'Ontario. $\mathrm{N}^{\circ}$ de catalogue En 153-39/1994F, 109 p.

G.E.O.D.E. (1994) "Dragage dans les ports français". Rapport de Synthèse (SOGREAH), septembre, $113 \mathrm{p}$.

G.E.O.D.E. (1996) "Bilan d'activité : suivi de la qualité des matériaux de dragage". $4 \mathrm{p}$.

Kamon M. et Nontananandh S. (1990) "Contribution of stainless steel slag to the development of strength for seabed hedoro". Japanese Society of Soil Mechanics and Foundation Engineering. Vol. 30, $n^{\circ} 4$, pp. 63-72

Knox D. P. et Najjar R. A (1994) "Solidification and stabilisation of lagoon sludge and dredged spoils". $1^{\text {st }}$ Congress on Environmental geotechnics, Alberta, Canada, Juillet 11-15, pp. 41-47

Ogino T., Goto T., Kataoka K. et Kuroda M. (1994) "Utilization of stabilized dredged waste for construction material". $I^{\text {st }}$ Congress on Environmental geotechnics, Alberta, Canada, Juillet 11-15, pp. 49-56

Sollars C. J. et Perry R. (1989) "Cement-based stabilisation of waste : practical and theoretical considerations". Journal of the Institute of Environment Management, Vol. 3, $N^{\circ} 2$, pp. 125-134

Spaque. (1998) "La gestion des matières issues du dragage et du curage des cours d'eau et la problématique des centres d'enfouissement technique". Société Publique d'Aide à la Qualité de l'Environnement, Liège, $33 \mathrm{p}$.

Stegemann J. A., Cote P.L. (1991) "Programme conjoint d'étude de méthodes d'essai pour l'évaluation des déchets solidifiés". Série de la protection de l'environnement SPE 3/HA/8, Environnement Canada, $131 \mathrm{p}$.

U.S.E.P.A (1998) "Information Sources for Innovative Remediation and Site Characterization Technologies". Edition électronique (CD-ROM) du Rapport EPA 542-C-98-003.

Van Leeuwen J., Pepels A. et Van Emst G. (1997) "Contaminated soil-cement stabilization in a demonstration project". ENG, Putting theory in a practice WASCON, Houthem St. Gerlach, NLD, 8 p. 